\title{
Temperature Rise Within Radioactive Liquid Wastes Injected Into Deep Formations
}

GEOLOGIGAL SURVEY PROFESSIONAL PAPER 386-A

Prepared in cooperation with the U.S. Atomic Energy Commission

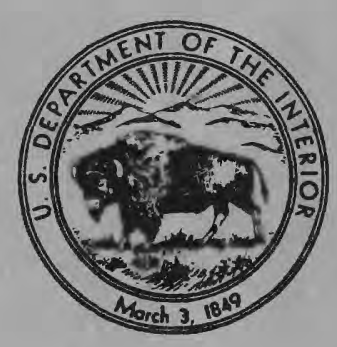




\section{Temperature Rise Within}

Radioactive Liquid Wastes

\section{Injected Into Deep}

Formations

By HERBERT E. SKIBITZKE

CONTRIBUTIONS TO PROBLEMS OF RADIOACTIVE WASTE DISPOSAL

GEOLOGICAL SURVEY PROFESSIONAL PAPER 386-A

Prepared in cooperation with the

U.S. Atomic Energy Commission 


\section{UNITED STATES DEPARTMENT OF THE INTERIOR \\ STEWART L. UDALL, Secretary \\ GEOLOGIGAL SURVEY \\ Thomas B. Nolan, Director}

For sale by the Superintendent of Documents, U.S. Government Printing Office Washington 25, D.C. - Price 15 cents (paper cover) 


\section{CONTENTS}

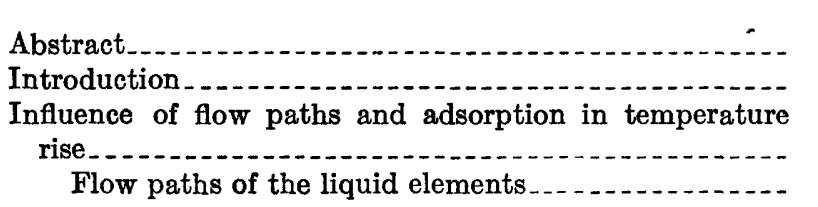

Page

A-1 Influence of flow paths, etc.-Continued

1 Concentration of thermal energy by adsorption process..........

1 Other parameters controlling temperature rise

1 References....

Page

\section{ILLUSTRATIONS}

Figure 1-Diagram showing flow lines of liquid waste being introduced into a homogeneous and isotropic aquifer through an injection well.-

2-Diagram showing effects of adsorption on flow of wastes away from well.

3-Approximate temperature rise in 3,650 days per gram of fission products per gallon of

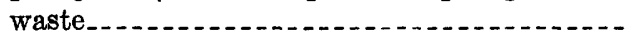

Page

FIGURE 4-Approximate amount of fission products, in grams per gallon of waste, producing a A-2 temperature rise of $100^{\circ} \mathrm{F}$, for different

3 cooling periods..... A-6

5
5 -Graphs illustrating variations of erf $\frac{A}{2 \sqrt{k\left(t-t^{\prime}\right)}}$ with thickness, thermal diffusivity of aquifer, and cooling period...-..-..-
Page

\section{TABLES}


, 


\title{
TEMPERATURE RISE WITHIN RADIOACTIVE LIQUID WASTES INJECTED INTO DEEP FORMATIONS
}

\author{
By Herbert E. Skibitzke
}

\begin{abstract}
The data and method of analysis presented here provide for an approximate determination of expected temperature rises. They show that, in general, when large masses of liquid waste are injected into an aquifer through wells, heat conduction is not very significant, and as an approximation the temperature rise within the aquifer, since injection began, is equal to the

total heat generated in a unit volume of aquifer divided by the product of the specific gravity of the aquifer and contained waste and its heat capacity. The generalizations outlined here should lead to an understanding of the relative importance of heat storage as compared to thermal conduction away from the region.
\end{abstract}

\section{INTRODUCTION}

The operation of a nuclear reactor results in the fission of the nucleus of heavy atoms into atomic particles of smaller mass. The resulting particles, or fission products, inhibit the fission process; consequently, they are periodically removed from the reactor. These particles, together with the solvent and other material in solution, constitute the radioactive wastes under discussion.

Because of radiation, the radioactive wastes are dangerous and must be handled accordingly. The fission products in the wastes start decaying from the time they are produced; energy is released in the form of beta particles $(\beta)$ and gamma rays $(\gamma)$, which produce heat. In considering the disposal of these wastes by injecting them through wells into the earth, the amount of heat they release is of considerable importance, for, in general, earth material is a poor thermal conductor.

The temperature rise within earth material due to the injection of heat-emitting radioactive liquid wastes is difficult to determine analytically. Because of the complexity of the boundary conditions involved, the temperature rise can be determined only by elaborate numerical analysis. It is possible, however, to establish a simple relationship, which is valid for certain conditions, and from this relationship to determine the rise in temperature. The relationship to be established is that thermal conduction is unimportant with relation to heat storage for the conditions outlined here.

\section{INFLUENCE OF FLOW PATHS AND ADSORPTION IN TEMPERATURE RISE}

The total rate of heat generation from a given quantity of fission products has been calculated from theory and determined by experiment. The results have been described by many authors, such as Coryell (1947); Coryell and Sugarman (1951); and Culler (1955).

The factors controlling the rate of heat transmission in an aquifer away from an injection well are the geometrical configuration formed by the heat-emitting waste material, the paths of heat conduction away from that material, and the thermal diffusivity of the saturated porous medium. The shape of that portion of the aquifer occupied by the injected waste changes in a complex manner with time and other factors and thus is difficult to define analytically. The establishment of the principles involved requires some simplifying approximations from which the main features of those principles may be derived. First, however, it is desirable to describe the dynamics of the flow of waste away from the site of injection. With the exception of liquid viscosity the flow of the waste is controlled, in general, by parameters independent of temperature.

\section{FLOW PATHS OF THE LIQUID ELEMENTS}

The flow lines for a liquid entering a homogeneous and isotropic aquifer from an injection well are shown in figure 1 . The flow lines are the result of compounding the radial flow from the well with the linear flow that existed in the aquifer prior to the injection of the liquid waste; thus, the flow system shown in figure 1 is linearly homogeneous. The maximum width to be reached by the radioactive waste is shown in figure 1 by the dashed lines $A A^{\prime}$ and $B B^{\prime}$. This width, which may serve as a scale factor, is proportional to the rate of injection divided by the flow per unit strip of aquifer. 


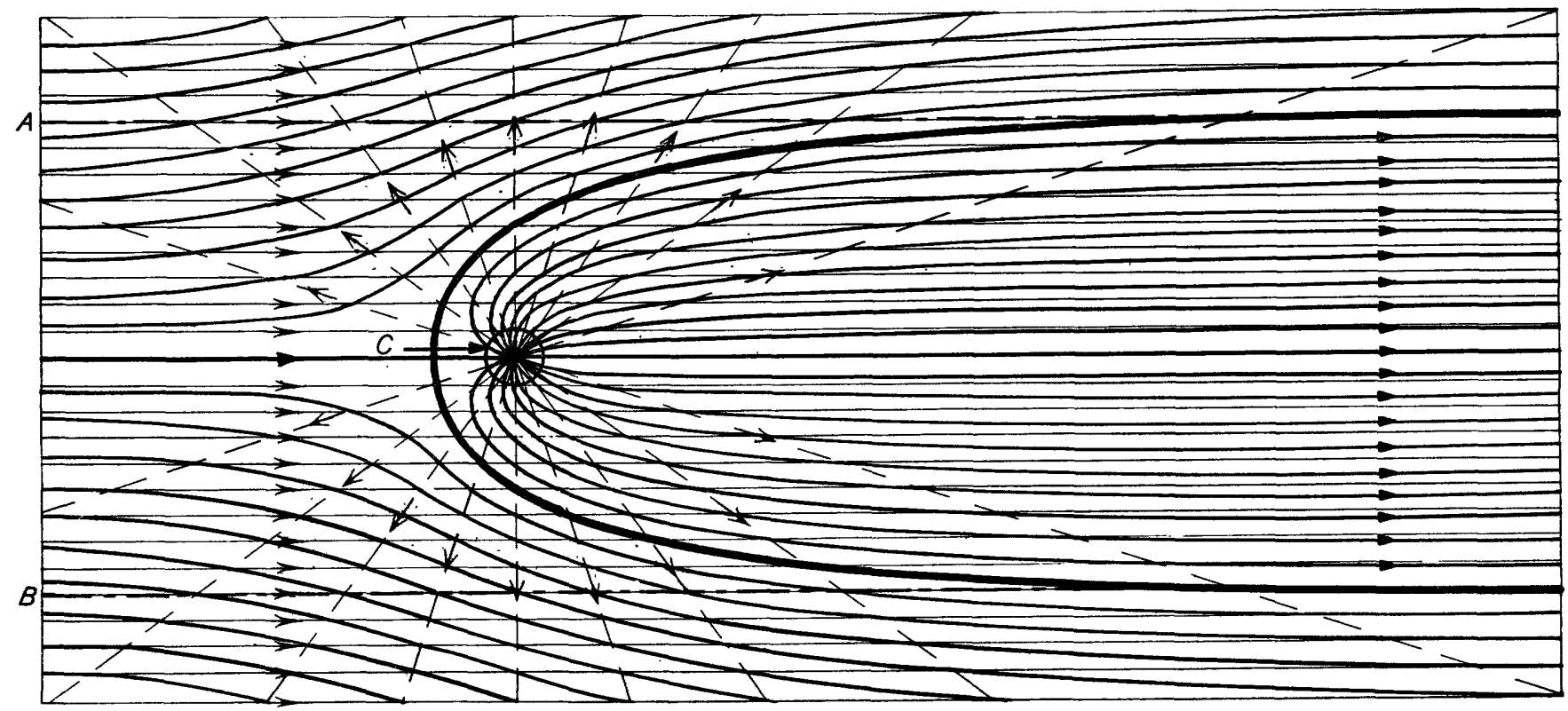

Flow lines of ground water moving in the aquifer before injection starts

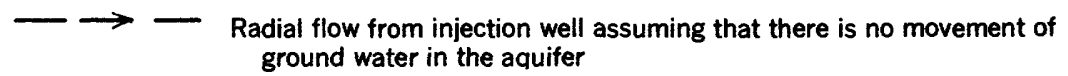
ground water in the aquifer

Flow lines resulting from injection of waste in a system of regional ground-water flow

FIGURE 1.-Diagram showing flow lines of liquid waste being introduced into a homogeneous and isotropic aquifer through an injection well.

Many deep-lying aquifers have a low transmissibility, resulting in low flow rates. The width of the contaminated zone in such aquifers could thus be very large; for example, an injection rate of 10,000 gallons a day could give a maximum width of several thousand feet in an aquifer having a transmissibility of a few hundred gallons a day per foot. For such an injection rate that part of the flow system within the circle lettered " $C$ " in figure 1 would be largely radial and is the most significant. The region " $C$ " would be the region in which the contaminant would be confined by adsorption.

The movement of the fission products and other solutes in the waste does not necessarily coincide with that of the solvent. The rate of motion of the dissolved matter is retarded, as the solute spends a portion of its time in transit adsorbed on the solid surfaces of the porous medium after the waste is injected. The solvent, however, is not adsorbed; consequently, the solute moves at a slower rate. The adsorption process depends upon the chemical nature of the dissolved matter as well as upon the chemical nature of the surface of the porous medium. Although it is not the purpose of this discussion to analyze in detail the effects or process of adsorption, it is of significance to consider in a general way the effects of adsorption on retardation of the motion of the waste.

With relation to adsorption within the radial-flow system three different situations may appear: (a) no adsorption, (b) adsorption uniform for all materials, and (c) nonuniform adsorption; these are represented schematically in figure 2 . The most strongly adsorbed materials will tend to occupy most of the available absorption sites, thereby permitting the less strongly adsorbed ions to move on. Because some of the strongly adsorbed materials are fixed temporarily by adsorption, these materials move most slowly. This allows the weakly adsorbed components to move to a part of the aquifer where adsorption sites are available and where they become adsorbed. Thus, a selective banding of differing chemicals would be produced circumferentially about the well.

The first illustration in figure 2 , case $A$, represents a condition in which no adsorption occurs and the solute moves outward at the same rate as the solvent. This is an idealized condition that would not occur in reality. 
In case $B$ all solutes are assumed to be adsorbed at the same rate and the solvent moves outward at a faster rate than the solutes. This second situation also is idealized and probably would not occur in reality. The third illustration, case $C$, represents a multicomponent system wherein one of the components is strongly adsorbed whereas the remainder of the components are less strongly adsorbed. With respect to the disposal of reactor wastes, the aluminum nitrate common to those wastes would be more strongly adsorbed then the fission products; consequently a large cylinder of adsorbed aluminum nitrate would occur close to the well. There could be little adsorption of fission products within this cylinder; then they would move through the cylinder into an uncontaminated part of the aquifer where they would be adsorbed. The solvent would move outward beyond the area occupied by the fission products. This third condition is more like the actual conditions; moreover, further complications would occur because of the large number of different elements present, which are adsorbed at different rates, and also because of anisotropy and nonhomogeneity of the porous medium. The saturated sediments would behave as a chromatographic column; hence, a series of rings corresponding to different fission products and other solutes would occur. The illustrations shown in figure 2 depict, in simplified form, the configuration of those parts of the aquifer occupied by heat-generating material that must be described analytically in order to determine the temperature rise due to radioactivity. The analytic representations so derived become the boundary conditions for the differential equations for heat conduction. The solution of such differential equations in closed form is very difficult; however, certain limiting approximations illustrate the effects of the various factors controlling the temperature rises.

\section{CONCENTRATION OF THERMAI ENERGY BY ADSORPTION PROCESSES}

The amount of heat produced by fission products is proportional to the amount of $\beta$ particles, $\gamma$ radiation and the respective energies that they release during their decay. The rate of heat production can be computed from the sum of the energies emitted by the different fission products or can be computed approximately by considering the fission products as a statistical assembly. The first method is, of course, more precise, especially after the short-lived products have decayed completely.

Experimental data furnished by various authors (see Coryell and Sugarman, 1951, p. 457) show that the total energy released by the fission products, and thus the total heat produced, follow an exponential law of the form $C t^{-1.0}$ to $C^{\prime} t^{-1.41}$ depending on the cooling period. Where $C$ and $C^{\prime}$ depend upon units of power desired.

An exponential law representing the energy release after a cooling period of a year probably would be of the

$$
C_{1} \times 10^{3} \times t^{-1.35} \frac{\text { Watts }}{\text { Grams of fission products }}
$$

form where $C_{1}$ is the number of Watts per Gram being emitted in one day. This is the law corresponding to the longest cooling period recorded, 16 to 340 days, and probably would be valid for a period of about 6 or 7 years. (From the experimental data of Brady and Turkevich, quoted by Coryell and Sugarman, 1951, p. 457).

The heat emission per unit volume of aquifer would be higher near the well for case $A$ (fig. 2) because the waste in the outer region would have a longer time to decay, the magnitude of the effect being controlled by the time of travel and the exponential law governing the heat release. If the same amount of heat-generating

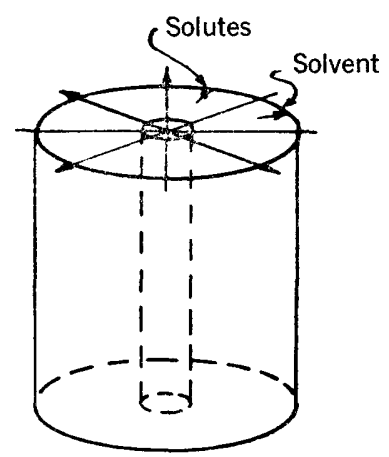

Case $A$

Homogeneous distribution of solute within the solvent; no adsorption

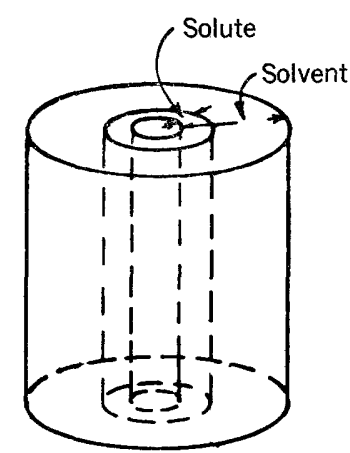

Case $B$

Nonhomogeneous distribution of solute within the solvent; uniform adsorption
Fission products arid $\begin{aligned} \text { Aluminum byproducts } & \text { other less strongly } \\ \text { and other strongly } & \text { adsorbed solutes }\end{aligned}$ adsorbed solutes

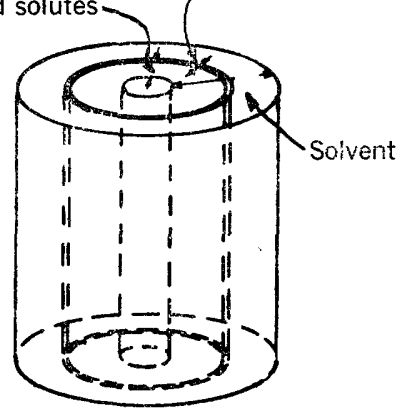

Case $C$

Nonhomogeneous distribution of solute within the solvent; nonuniform adsorption 
material per unit volume of injected liquid were involved in case $B$ as in case $A$, the heat emission per unit volume in the central cylinder would be higher in case $B$. Case $C$ is more complex because the fission products traveling from the well to the cylinder of fission products would have the least time to decay. However, the concentration of heat produced per unit volume in the inner cylinder would be relatively low because the adsorption process concentrates the material emitting heat in the outer cylinder and therefore the total heat being emitted per unit volume in the cylinder of fission products would be substantially higher.

\section{OTHER PARAMETERS CONTROLLING TEMPERATURE RISE}

The factors controlling temperature rise, other than the rate of heat emission by the fission products, are the geometry of the heat-emitting zone, the thermal diffusivity of the aquifer, and the temperature of the injected water. In case $A$, the effects of the temperature of the injected water have importance only in the sense of setting the allowable temperature rise before the liquid changes to vapor. In cases $B$ and $C$, the injected water flows through that part of the aquifer occupied by heat-emitting material and thereby could have a cooling effect.

The remaining factors of geometry and thermal diffusivity may be determined for the simple condition condition shown in case $A$, wherein the flow has ceased and heat emission continues. The relative importance of various effects may be determined for case $A$ and from these data the effects for cases $B$ and $C$ may be evaluated.

The heat emission from a region, such as that described by case $A$, can be determined by Fourier's method (Carslaw and Jaeger, 1947). This method permits the determination of the temperature rise at only one point in the heat-conduction system under study. For the problem under consideration, the temperature rise at the center of the cylinder containing waste- that is, at the injection well-would be the greatest and therefore the most critical. This point is chosen as the center of the coordinate system in the following analysis. The temperature at this point may be determined by summing the effects of all infinitesimal elements emitting heat. The effect on the coordinate center owing to an infinitesimal element at $r^{\prime}, \theta^{\prime}, z^{\prime}$ at time $t$ for heat emitted at time $t^{\prime}$ would be (Carslaw, p. 218)

$$
\Delta V=\frac{\beta\left(r^{\prime} \theta^{\prime}, z^{\prime} t\right) r^{\prime} d r^{\prime} d \theta^{\prime} d z^{\prime} d t^{\prime}}{8 \rho c\left[\pi k\left(t-t^{\prime}\right)\right]^{3 / 2}} e^{-\frac{\left(r^{\prime 2}+z^{\prime 2}\right)}{4 k\left(t-t^{\prime}\right)}}
$$

Where

$$
\begin{aligned}
\beta\left(r^{\prime}, \theta^{\prime}, z^{\prime}, t^{\prime}\right)= & \text { rate of heat emission in the infin- } \\
& \text { itesimal element } \\
\Delta V= & \text { temperature increase at the coordi- } \\
& \text { nate center } \\
r^{\prime}= & \text { radial distance from origin about the } \\
& z \text { axis } \\
t^{\prime}= & \text { time of emission of a given quantity } \\
& \text { of heat } \\
\theta^{\prime}= & \text { angular distance from origin about } \\
& \text { the } z \text { axis } \\
z^{\prime}= & \text { distance above the origin along the } \\
& z \text { axis } \\
t= & \text { time at which the temperature is to be } \\
& \text { determined } \\
\rho= & \text { specific gravity of the aquifer and } \\
& \text { liquid waste combined } \\
c= & \text { heat capacity of the aquifer material } \\
& \text { and contained waste } \\
k= & \text { thermal diffusivity of the aquifer }
\end{aligned}
$$

The summation of all such elements would be

$$
V=\int_{0}^{t} \int_{0}^{A} \int_{0}^{a} \int_{0}^{2 \pi} \frac{2 \beta\left(r^{\prime}, \theta^{\prime}, z^{\prime}, t^{\prime}\right) r^{\prime} e^{-\frac{\left(r^{\prime 2}+z^{\prime 2}\right)}{4 k\left(t-t^{\prime}\right)}}}{8 \rho c\left(\pi k\left(t-t^{\prime}\right)\right)^{3 / 2}}
$$

Where

$$
\cdot d t^{\prime} d z^{\prime} d r^{\prime} d \theta^{\prime}
$$

$A=$ half the thickness of the heat-emitting cylinder of wastes

$a=$ the radius of the heat-emitting cylinder of wastes

$V=$ temperature increase after a period of time $\left(t-t^{\prime}\right)$

The first three integrations may be made by assuming that the waste has spread much farther radially than the vertical thickness of the invaded cylinder, and that the geometrical distribution of heat emission is of no significance so that each element is emitting heat at the same rate throughout the cylinder. With these limitations in mind, the integration of (2) yields

$$
V=\int_{0}^{t} \frac{\beta(t)}{\rho c} \operatorname{erf} \frac{A}{2 \sqrt{k\left(t-t^{\prime}\right)}} d t^{\prime}
$$

As further integration would be possible only by numerical methods, which would further complicate the problem, an approximation is necessary to put the integral into closed form. The approximation is that

$$
\operatorname{erf} \frac{A}{2 \sqrt{k\left(t-t^{\prime}\right)}}
$$

is roughly equal to unity for most values of $A$ and $k$ 
throughout times of interest and for the type of aquifers likely to be used as waste disposal sites. When this is valid, then

$$
V=\int_{0}^{t} \frac{\beta(t)}{\rho c} d t^{\prime}
$$

Equation (4) states simply that the temperature rise at the center of that part of the aquifer invaded by the waste is the total of all heat emitted in a unit volume at the center, since time zero, divided by the product of the heat capacity of the aquifer and contamed waste and its specific gravity.

This conclusion could be arrived at qualitatively from analyzing the temperature response at the center of the cylinder. At time $t=0$ the temperature rises in each element at the same rate. Thus, all the elements start to emit heat at the same rate, except for the quantity of heat being transported away by conduction. The transport of heat by conduction requires a thermal gradient to exist. The gradient appears at the boundary instantly, but at the center all the surrounding elements are rising in temperature at the same rate. Therefore, the thermal gradient migrates inward at a very slow rate because of the low thermal diffusivity of earth material. The effects of migration of the thermal gradient into the center of the contaminated mass are represented by the erf term of equation (3). The approximation made depends on this erf term being essentially unity. The validity of this approximation is considered later in this paper; at this point, however, equation (4) is assumed to be valid.

The maximum temperature rise at the center of the contaminated zone may be determined by computing the total amount of heat emitted in a unit volume of aquifer and dividing it by the product of its specific gravity and its heat capacity. As an example of this effect, it will be assumed that a mass of 1 gram of gross fission products is dissolved in each gallon of waste and that the fission products have cooled for 1 year prior to injection into a sandstone aquifer having a porosity of 20 percent and a factor of heat capacity times specific gravity of 30 . Assuming that the -1.35 decay law holds, the total heat emitted per cubic foot of aquifer in 10 years will be about 30,000 BTU. This divided by the specific heat per unit volume corresponds to a temperature rise of $1,000 \mathrm{de}-$ grees Fahrenheit. The effect of different waste-cooling periods on the value of the temperature rise is shown in figure 3 . The maximum temperature rise before vaporization occurs determines the maximum concentration allowable in grams of fission product

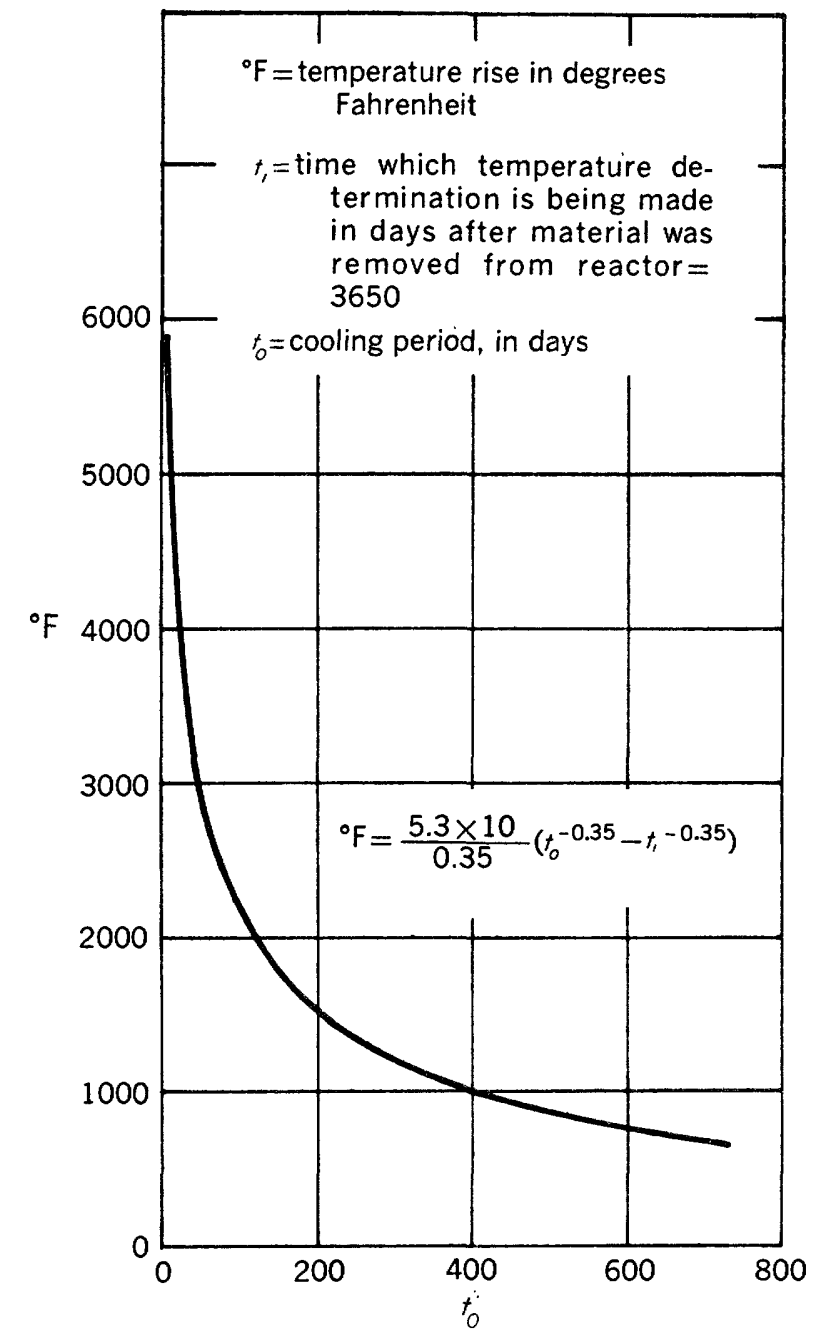

Figure 3.-Approximate temperature rise in 3,650 days per gram of fission products per gallon of waste.

per cubic foot of aquifer if the wastes are to remain in liquid form in the aquifer. The concentrations allowable for a $100^{\circ} \mathrm{F}$. rise of temperature for various cooling periods are shown in figure 4 . The response for a different maximum temperature is directly proportional to the ratio of that temperature to $100^{\circ} \mathrm{F}$.

The foregoing data are based on the approximation of the function erf $\frac{A}{2 \sqrt{k}\left(t-t^{\prime}\right)}$ being equal to unity. The function is dependent upon A, the half thickness of the aquifer; $k$, the thermal diffusivity of the aquifer; and the factors $t^{\prime}$, the time when heat emission begins, and $t$, the time for which the temperature is to be determined.

The effects of these variables on the value of the erf are shown in the graphs of figure 5. Each of the three 


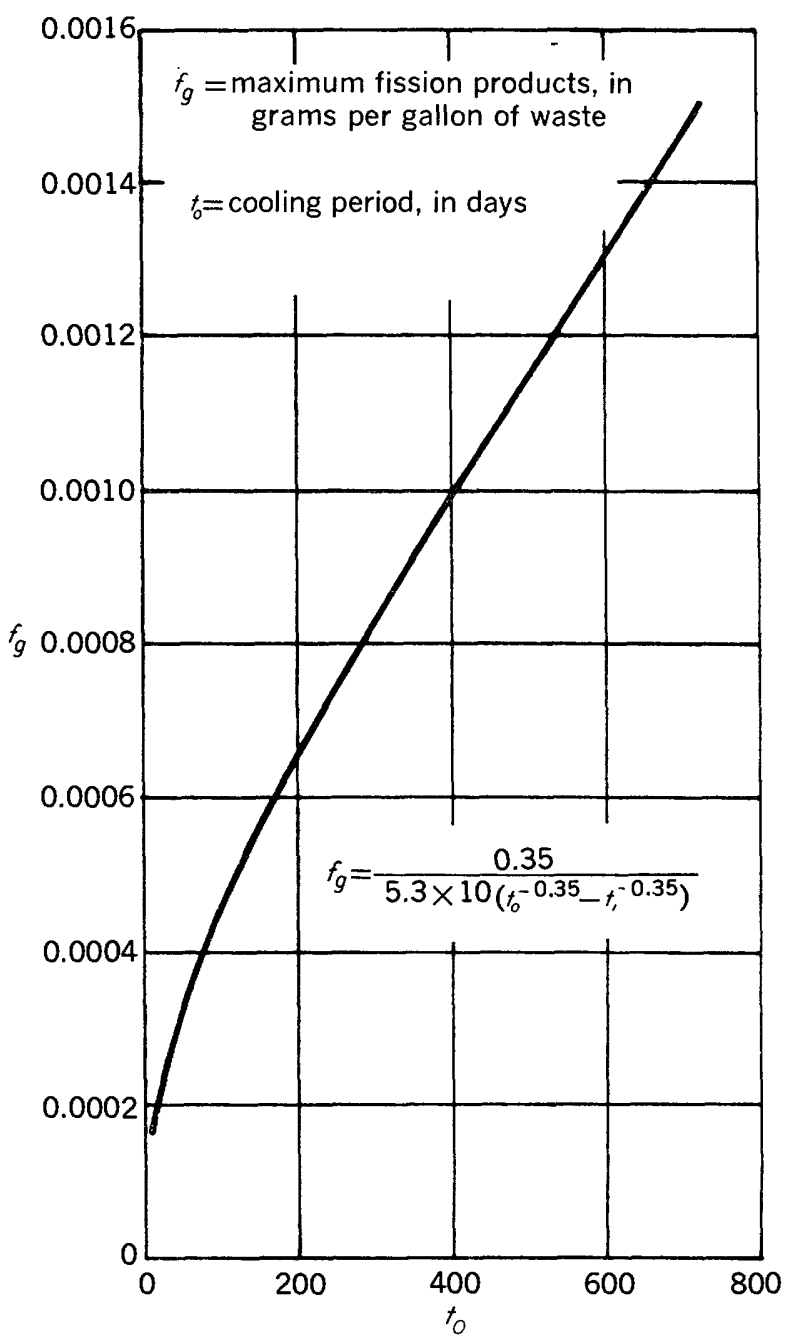

FigoRe 4.-Approximate amount of fission products, in grams per gallon of waste, producing a temperature rise of $100^{\circ} \mathrm{F}$, for different cooling periods.

columns of graphs corresponds to a different value of $A$. The first column is for $A=5$-that is, for an aquifer 10 feet thick. The second column is for $A=50$, for an aquifer 100 feet thick, and the last column is for $A$ $=100$, for an aquifer 200 feet thick. The test of the validity of the approximation made is the closeness with which the function approaches a value of unity--a straight line along the top of the graph. The graphs are arranged vertically so that the shortest cooling period ( 365 days) is in the uppermost graphs. The next lower set of graphs is for a cooling period of 10 years and the bottom set for a period of 30 years. Each series of graphs has three curves, corresponding to different thermal diffusivities. The uppermost curve is for a thermal diffusivity of $0.02 \mathrm{ft}^{2}$ per day, the next lower for a thermal diffusivity of $0.2 \mathrm{ft}^{2}$ per day, and the bottom curve for a thermal diffusivity of $0.94 \mathrm{ft}^{2}$ per day. These data show that only one curve of the first group (aquifer thickness 10 feet) approaches the approximation used here. This curve is for a small coefficient of thermal diffusivity (poor conductivity) and a short peroid of time. For other curves, representing higher thermal diffusivities, the thermal gradients have time to move through the short distance $A=5$ feet, and thus conduction in these conditions is of large significance.

The second column of graphs, for aquifers 100 feet thick ( $A=50$ feet), represents conditions greatly different from those expressed by the graphs in the first column. The upper graph, which represents a cooling time of 1 year, shows that the approximation is valid, being almost exact for all the three values of thermal diffusivity. The next lower set of curves (for a 10-year cooling period) shows that for all except the largst coefficient of thermal diffusivity, the approximation is very nearly exact; such a diffusivity, however, probably is to be expected in many consolidated sandstones. The graph shows that the approximation is, in general, more than 50 percent correct even for the largest thermal diffusivity. Because, at present, it is of interest to establish the order of magnitude of the problem, the curve for the highest thermal diffusivity may be considered a satisfactory approximation for as much as 10 years after heat emission begins. The bottom graph shows that even for a 30-year cooling period the approximation is satisfactory except for the highest thermal diffusivity.

The third column of graphs, which is for an aquifer 200 feet thick $(A=100)$, shows that the approximation is essentially valid for cooling periods of 1 year and 10 years for all the thermal diffusivities considered and the approximation is valid also for a cooling period of 30 years except for the highest thermal diffusivity. Even for that diffusivity, however, the approximation is more than 50 percent valid.

The graphs in figure 5 illustrate that the effect of conduction is felt much earlier in thin aquifers because of the lesser distance for the thermal gradient to migrate and also that the effect of conduction is felt quicker for larger thermal diffusivities. The thermal diffusivity of various earth materials is shown in table 1 . The conclusion may be drawn that for thick sandstone aquifers having a thermal diffusivity of about 0.94 (calculated from the thermal-diffusivity value of 1.0 given for sandstone, table 1 , and corrected for water saturation), the approximation is satisfactory with respect to a functional relationship. However the question of geometric distribution of wastes due to flow and adsorption conditions still remains.

It has been assumed that the liquid has moved radially for greater distances than the aquifer half-thickness $A$. The amount of water required to accomplish this would be as follows: 
A cylinder of an aquifer having a thickness of 10 feet $(A=5)$, a radius of 5 feet, and a porosity of 20 percent contains about 1,200 gallons of liquid. If the aquifer were 100 feet thick and the radius of the cylinder 50 feet, the cylinder would contain about $1,200,000$ gallons of liquid; and if the aquifer were 200 feet thick, and the radius 100 feet, it would contain about 8 times this latter amount. If there were less than these amounts of liquid for any of the above aquifer thicknesses, the calculated temperature rises would be too large for the long periods of time.

Because of adsorption the dimensions of the contaminated body would decrease, but the amount of heat being emitted per unit volume would increase. These two effects tend to cancel one another.

The effects of decay of thermal energy released per unit of time would be to institute a small thermal gradient radially away from the well. The magnitude of this effect is dependent upon the rate of growth of the cylindrical volume away from the well. If large quantities were injected, so that the growth would be rapid,
TABLE 1.-Thermal properties of some common substances [Adapted from Carslaw and Jaeger, 1947, p. 382]

\begin{tabular}{|c|c|c|c|c|}
\hline Substance & $\begin{array}{l}\text { Specific } \\
\text { gravity } \\
(\rho) \mathrm{lb} / \mathrm{ft}^{3}\end{array}$ & $\begin{array}{l}\text { Heat } \\
\text { capac- } \\
\text { ity }(c) \\
\text { Btu/lb } \\
{ }^{\circ} \mathbf{F}\end{array}$ & $\begin{array}{l}\text { Thermal } \\
\text { Conduc- } \\
\text { tivity } \\
(K) \\
\text { Btu } \\
\left(\text { day } \mathrm{ft}^{3}\right) \\
\left({ }^{\circ} \mathrm{F} / \mathrm{ft}^{3}\right)\end{array}$ & $\begin{array}{l}\text { Thermal } \\
\text { Diffu- } \\
\text { sivity } \\
\mathrm{ft}^{2} / \text { day }^{1}\end{array}$ \\
\hline Air & 0.08 & 0.24 & 0.34 & 17. 5 \\
\hline Granite & 162. & .21 & 35. & 1. 0 \\
\hline Limestone & 156. & .22 & 23. & .65 \\
\hline Sandstone & 143. & .23 & 35. & 1.0 \\
\hline Average rock & $--\infty$ & $-\cdots$ & 24. & 1. 1 \\
\hline Ice_-_- & 57. & .50 & 31. & 1. 1 \\
\hline Concrete $(1: 2: 4)$ & 144. & .23 & 13. & .39 \\
\hline Brick (building) & 162. & .20 & 12. & .35 \\
\hline Soil (average) & 156. & .2 & 14. & .43 \\
\hline Soil (sandy, dry) & 103. & .19 & 3. 7 & .19 \\
\hline $\begin{array}{l}\text { Soil (sandy, moisture con- } \\
\text { tent } 8 \text { percent) }\end{array}$ & 109. & .24 & 8. 23 & .31 \\
\hline Water & 62.5 & 1. 0 & 8. 5 & .13 \\
\hline
\end{tabular}

1 Values computed from the table values for $\rho, c$, and $K$.
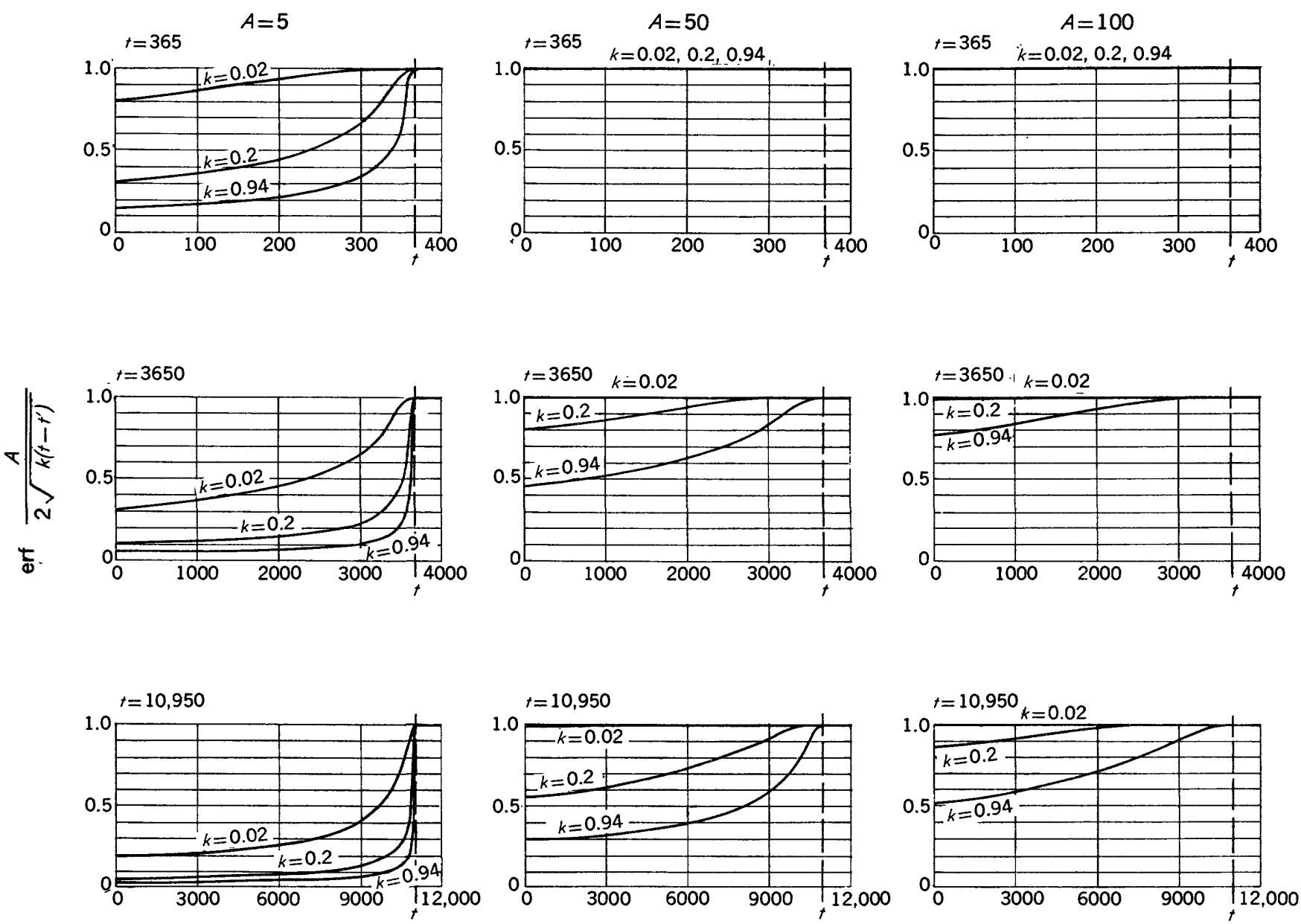

$t^{\prime}$ in days

FiguRe 5.-Graphs illustrating variations of $\operatorname{erf} \frac{A}{2 \sqrt{k}\left(t-t^{\prime}\right)}$ with thickness, thermal diffusivity of aauifer, and cooling period. 
this effect would be greatly reduced and there would be little or no thermal gradient.

With respect to hydraulics, the dynamic state of the region invaded by the waste would further modify the preceding conditions because of the cooling effect caused by the injected liquid flowing through the contaminated region, and by the porous solid which absorbs some heat. When injection ceases, these modifying effects lose their importance.

\section{REFERENCES}

Carslaw, H. S., and Jaeger, J. C., 1947, Conduction of heat in solids: Clarendon, England, Oxford Press.

Coryell, C. D., and Sugarman, Nathan, 1951, Radiochemical studies: The fission products: New York, McGraw-Hill.

Coryell, C. D. and others, 1947, The science and engineering of nuclear power, Book 1: Cambridge, Mass., AddisonWesley Press.

Culler, F. L., Jr., 1955, Notes on fission product wastes from proposed power reactors: Oak Ridge National Laboratory ORNL Rept. no. 55-4-25. 